

\title{
Bullying and malocclusion in adolescence: a case report
}

\author{
Rossana Patricia Rotolo*; Ludovica Nucci* ; Vincenzo Grassia* ; Letizia Perillo*; Fabrizia d'Apuzzo \\ * Multidisciplinary Department of Medical-Surgical and Dental Specialties, University of Campania Luigi Vanvitelli, Naples, Italy
}

\begin{abstract}
Introduction: Adolescents with bad malocclusion can more often be victims of bullying and the improvement of their facial appearance through oral rehabilitation is nowadays an important issue. The aim of this case report was to describe the orthodontic treatment in a teenager with a dentoskeletal malocclusion exposed to bullying to correct his occlusal problems and improve facial esthetics and quality of life.

Case presentation: The patient, a boy aged 13.5 years, had a class II, division 1, malocclusion with hyperdivergent pattern, mandibular asymmetry, constricted maxillary arch and molar crossbite, increased overjet, lower mild crowding and lip sucking. A two-phase approach was necessary to achieve proper occlusion, better esthetics and promote the patient's self-esteem with consequent greater serenity of his family.

Conclusion: Treatment of adolescent patients with severe dento-skeletal malocclusions is among the most difficult challenges for orthodontists. Orthodontic therapy cannot simply aim a simple occlusal correction but also have to establish a valid methodology to remove the "cause" of bullying and to treat the patient, together with appropriate psychological support.
\end{abstract}

Ratolo RP, Nucci L, Grassia V, Perillo L, d'Apuzzo F. Bullying and malocclusion in adolescence:

a case report. South Eur J Orthod Dentofac Res. 2020;7(S1):17-20.

\section{INTRODUCTION}

Bullying is described as a behavioral phenomenon in which a fragile person, usually a teenager, is repeatedly exposed to intentional, verbal or physical, aggression by at least one other person. ${ }^{1}$

Bad actions against the victim may be direct (punches, kicks, insults and threats) or indirect (manipulation of social relationships with gossip or marginalization). ${ }^{2}$

Bullying is unfortunately very frequent among the school desks and its prevalence varies between $5 \%$ and $88 \%$ depending on location and age. ${ }^{3,4}$

Victims of bullying can suffer severe physical, mental and social

Corresponding Author:

Vincenzo Grassia

Multidisciplinary Department of

Medical-Surgical and Dental Specialties,

University of Campania Luigi Vanvitelli,

Via L. De Crecchio 6,

Naples 80138, Italy.

E-mail: grassiavincenzo@libero.it consequences such as anxiety, depression, sleep difficulties, low self-esteem, lower academic performance and isolation. ${ }^{5}$ The main targets, "cause" of the aggressions, are the physical characteristics, including mainly weight, height and facial appearance. Dental aesthetics are becoming increasingly important nowadays. ${ }^{6}$ People with well-aligned teeth and a beautiful smile are considered more attractive, intelligent and more suited to social life than those with malocclusion or misaligned teeth. Among the occlusal characteristics most common object of insults, we find the alterations of overjet and overbite, with the so-called "rabbit teeth," followed by crowding, diastemas, anomalies of shape and position of the teeth.

These occlusal characteristics, even more so if associated with marked skeletal modifications (severe class II, class III, asymmetries and divergence alterations), can cause derision and psycho-physical violence so affecting the general individual well-being in the society. ${ }^{7}$ Thus, the purpose of this paper is to report a clinical case of an adolescent patient willing to get an orthodontic treatment to improve his facial appearance after some episodes of bullying at school. 


\section{CASE REPORT}

The patient came to the Orthodontic Program of the University of Campania Luigi Vanvitelli in Naples, Italy, with their parents who were worried after their son had been injuring by some of his classmates due to his buckteeth and because he was deeply unhappy with his smile and face. He was 13 years and 5 months old Caucasian boy in the late mixed dentition phase. Class II, division 1, subdivision right, malocclusion with hyperdivergent pattern, mandibular asymmetry to the right due to longer left mandibular ramus; constricted maxillary arch, crossbite of 16 , increased overjet $(6 \mathrm{~mm})$, mild crowding, $1.5-\mathrm{mm}$ lower midline deviation to the right and lower lip sucking (Figure 1). The patient did not receive any previous dental or orthodontic treatment.
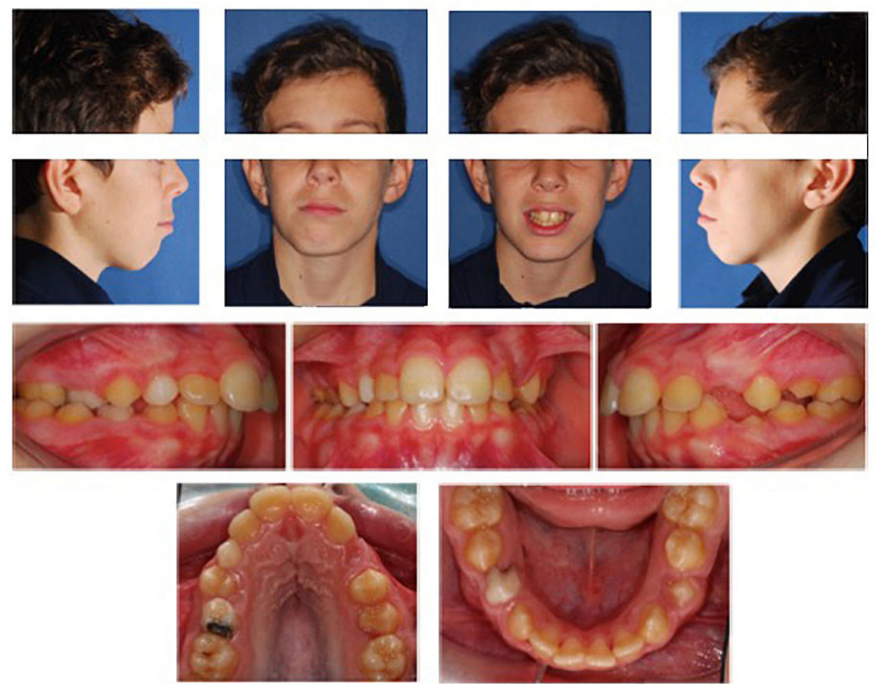

Figure 1. Pre-treatment records

About his medical history, it may be worthy of note the presence of mouth breathing, often caused by asthma attacks, and the diagnosis of primary sclerosing cholangitis. This pathology may cause several oral problems, like mucosal lesions, enamel defects and gingivitis, after eventual complications of the pathology, mainly in adulthood.

The patient had a correct posture of the body, head, neck and shoulders.

In the frontal view, the patient had an asymmetric face with mandibular deviation on the right side, increased anterior lower facial height, bipupillar line not parallel to the horizontal plane. In lateral view, the profile was convex with incompetent lips at rest. The nasolabial angle was within normal limits while the mentolabial angle was flattened. The patient did not show signs or symptoms of craniofacial pain, temporomandibular disorders. As bad habits, lower lip sucking was reported. There was no history of headache or trauma and no muscle pain, TMJ problems or CO-CR discrepancy were registered.
The oral hygiene was poor and tooth decays were present in 16 , 55 and 84 .

The panoramic radiograph showed that all the permanent teeth were present, but a lack of space for permanent canines was evident in the upper arch, mainly on the right side.

The cephalometric morphological assessment of the lateral skull radiograph showed a skeletal Class II $\left(\mathrm{A}-\mathrm{N}-\mathrm{Pg}=6^{\circ}\right)$ with hyperdivergency $\left(\mathrm{S}-\mathrm{N} / \mathrm{Go}-\mathrm{Gn}=45^{\circ}\right)$ and vertical growth pattern (Jarabak index $\mathrm{S}-\mathrm{Go} / \mathrm{N}-\mathrm{Me}=57 \%)$. The upper incisors were flared $\left(1-\right.$ ANS-PNS $\left.=119^{\circ}\right)$, whereas the lower incisors $\left(1-\mathrm{Go}-\mathrm{Gn}=90^{\circ}\right)$ showed normal values of inclination.

Overjet $(6 \mathrm{~mm})$ and overbite $(4 \mathrm{~mm})$ were increased but within normal values.

The postero-anterior skull radiograph evaluation revealed a maxillary contraction and showed a slight skeletal mandibular deviation on the right side due to a longer left mandibular ramus (Fig 2).

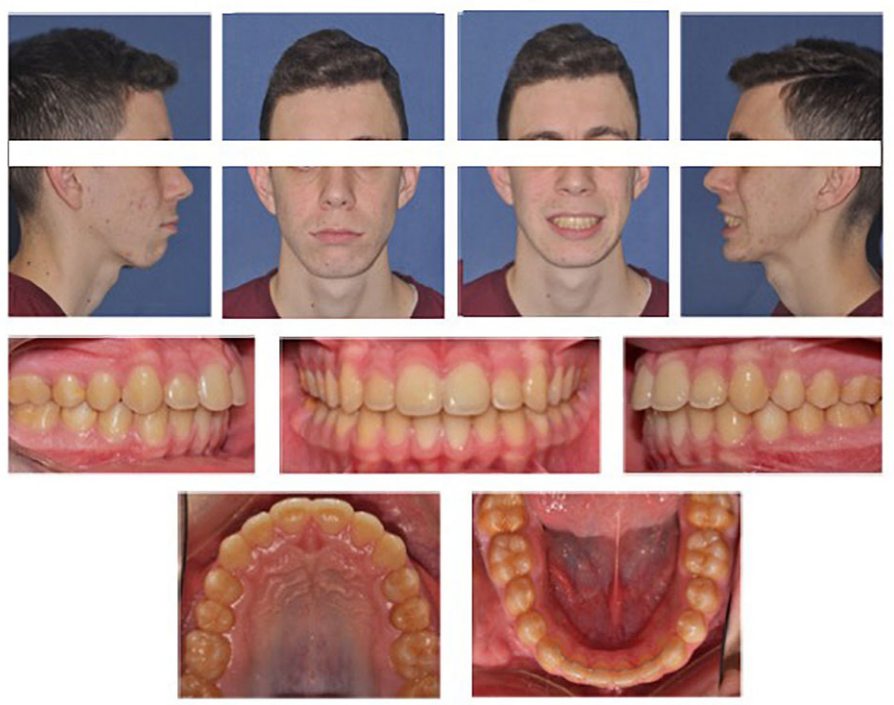

Figure 2. Post-treatment records

\section{Treatment objectives}

Based on the patient's age and diagnosis, the options were either to begin a two-phase orthodontic treatment or to wait and perform an orthodontic-surgical treatment in adult age. However, the two-phase orthodontic treatment chosen seemed to be the most rational option considering the patient and his parents' anxiety and expectations and the occlusal features showing maxillary constriction with unilateral crossbite and lack of space for the upper cuspids.

The main treatment objectives were maxillary expansion with crossbite correction, vertical growth pattern control, gain of space for upper cuspids, crowding correction, lower lip sucking control, alignment, leveling and arch form coordination, dental class and overjet correction. 
Thus, a two-phase orthodontic treatment was proposed and accepted by the patient and his parents after a conservative treatment of the upper right molar and the extraction of 55 and 84 .

In the first phase, the appliances used were a bonded McNamara maxillary expander for 9 months and a lip bumper on 36-46 in the lower arch for 10 months.

The patient underwent a reevaluation with interim records (both photographic and radiographic) just after the bonded expander removal.

In the second phase, the appliances used were a transpalatal arch applied on 16-26 at the initial stages of the fixed treatment, a 7-7 multibracket fixed appliance in the upper and lower arch (0.022x0.028" MBT prescription), Class II and cusp seating elastics. This phase of treatment lasted 2 years and 4 months.

The retention phase included a Hawley retainer appliance in the upper arch and a cuspid-to-cuspid fixed retainer in the lower arch.

\section{Treatment progress}

The treatment was performed by the same operator at the Orthodontic Program of the University of Campania Luigi Vanvitelli.

The first phase of treatment started one month later the initial check-up, in October 2013. Before starting the orthodontic treatment, the occlusal caries in 16 was treated while the decayed 55 and 84 were extracted, waiting for the permanent tooth eruption.

The McNamara expander was used to correct the maxillary constriction, to gain space trying to control the vertical growth pattern. It was bonded on the upper arch from canines to first molars bilaterally and the 11-mm expansion screw was activated until the crossbite was solved.

In the lower arch, bands were bonded on the first molars and a removable lip bumper was used both to reduce the lower crowding and to control the patient's oral habit of lower lip suction.

After 9 months, the McNamara expander was debonded and an interim reevaluation was performed in July 2014. Lower bands were left in situ to continue with the lip bumper treatment 1 month more during interim records analysis and discussion. To note, the patient was not compliant in wearing the lip bumper during the last months of the first phase of orthodontic treatment because of the death of his mother in that period.

The second phase of treatment started in August 2014 with a removable transpalatal arch applied on 16 and 26 to maintain the palatal expansion and to distally derotate the upper first molars. In September 2014, the upper and lower arches were fully bonded with $0.022 \times 0.025 "$ MBT multibracket fixed appliances. The following archwire sequencing was used: .016 nickel-titanium for alignment, .019x.025 nickel-titanium for leveling, .019x.025 stainless- steel for arch coordination and .018 AJ Wilcock Australian wire with refinement bends for the finishing stage. Class correction on the right side was achieved using Class II elastics applied on rectangular stainlesssteel archwires and cusp seating elastics were used during the finishing. During the treatment, the surgical removal of lower right third molar buds was required for the proximity to the 47 and thus to favor its correct eruption in the lower arch, as clearly showed by the interim panoramic radiograph. The patient was repeatedly reminded of the need to maintain accurate oral hygiene to avoid any gingival problem, and he was compliant enough, mainly during the second phase of therapy.

\section{Treatment results}

The treatment goals were achieved. The occlusal, functional and esthetic results were satisfactory, the patient and his family were happy of his smile. Although the patient's face in frontal view maintained a mandibular asymmetry on the right side, the smile arch was improved with no buccal corridors. The profile appears more harmonic and slightly improved (mainly thanks to the mandibular growth during the pubertal peak).

Oral hygiene during orthodontic treatment was quite good, periodontal tissues were healthy. There were no decayed elements or signs of enamel decalcification and the panoramic radiograph did not show any sign of bone loss or root resorption. Intra-oral photographs and dental casts showed a good alignment of marginal ridges, leveling and arch coordination was achieved: the crossbite and the dental Class II relationship on the right side were corrected while the dental Class I on the left side was maintained; the overjet was reduced and the dental midline deviation was corrected.

The final static occlusion was satisfactory also on the lingual side and no prematurities were present during protrusive and lateral mandibular movements. A panoramic radiograph revealed that good roots angulation was achieved (Fig 2).

The lateral skull radiograph showed the improvement of sagittal and vertical skeletal relations between pre-treatment and posttreatment cephalograms and the correction of dental class on the right side. However, the hyperdivergent growth pattern was not favorable, although the use of McNamara expander in the first phase and careful biomechanics with the multibracket fixed appliances. The slight skeletal mandibular deviation was not considered in the treatment plan being not a complaint for both the patient and his parents. 
The panoramic radiograph showed no signs of condylar resorption or periodontal disease. The third molars, except for the extracted 48, were present and impacted within the jawbones: 18 and 28 had formed while the 38 was a bud and the patient was sent to the oral surgeon to remove it for its proximity to the 37 . The upper right first molar was filled before starting the orthodontic treatment. No clear signs of root resorption can be noted. Root angulations were parallel.

The final lateral cephalogram assessment showed an improved sagittal jaw relation (A-N-Pg from $6^{\circ}$ to $4^{\circ}$ ). The hyperdivergent pattern was controlled (S-N/Go-Gn from $45^{\circ}$ to $44^{\circ}$ ) and the maxillary inclination was reduced.

The upper incisor inclination was slightly improved while the lower incisors maintained their inclination, comparing initial and final cephalograms (Fig 1).

Overjet and overbite have remained relatively unchanged.

\section{DISCUSSION}

The treatment goals were achieved. The occlusal, functional, esthetic and psychological results were satisfactory. ${ }^{8}$ The outcome was rewarding for the clinicians and appreciated by the patient and his parents.
The key points determining the treatment's success were good interdisciplinary cooperation (orthodontist and mental health specialist) and the parent's and patient's collaboration. It was important to begin the orthodontic treatment as soon as possible to improve the quality of the patient's present and future life.

Written informed consent was obtained from the patient's father for publication of this case report and accompanying images.

\section{CONCLUSIONS}

Treatment of adolescent patients with severe dento-skeletal malocclusions is among the most difficult challenges for orthodontists. In fact, orthodontic therapy cannot simply aim a simple occlusal correction but also have to establish a valid methodology to remove the "cause" of bullying and to treat the patient, together with appropriate psychological support, if necessary, to restore the dental and mental smile he has lost.

\section{CONFLICT OF INTEREST}

The authors of the present article declare no conflicts of interest.

\section{REFERENCES}

1. Esteban ANP, Contreras CCT, Rodríguez SPO, Aldana MSC, Bueno LMD, Silva BADPN. Bullying in adolescents: role, type of violence and determinants. Rev Esc Enferm USP. 2020;54:e03625.

2. Chan A, Antoun JS, Morgaine KC, Farella M. Accounts of bullying on Twitter in relation to dentofacial features and orthodontic treatment. J Oral Rehabil. 2017;44(4):244-50.

3. Scheffel DLS, Jeremias F, Fratelli CMB, Dos Santos-Pinto LAM, Hebling J, de Oliveira OB Jr. Esthetic dental anomalies as motive for bullying in schoolchildren. Eur J Dent. 2014;8(1):124-8.

4. Tristâo et al. Is there a relationship between malocclusion and bullying? A systematic review. Prog Orth. 2020;21:26.
5. Artese F. The orthodontist's reach in bullying. Dental Press J Orthod 2020;24(2):15 16.

6. DiBiase AT and Sandler PJ. Malocclusion, orthodontics and bullying. Dent Update 2001;28(9):464-6.

7. Seehra J, Newton JT, DiBiase AT. Bullying in school children - its relationship to dental appearance and psychosocial implications: an update for GDPs. Br Dent J. 2011;210(9):411-5.

8. Al-Omari IK, Al-Bitar ZB, Sonbol HN, Al-Ahmad HT, Cunningham SJ, Al-Omiri M. Impact of bullying due to dentofacial features on oral health-related quality of life. Am J Orthod Dentofacial Orthop. 2014;146(6):734-9. 